\title{
$k$-DIMENSIONAL REGULARITY CLASSIFICATIONS FOR $s$-FRACTALS
}

\author{
MIGUEL ANGEL MARTíN AND PERTTI MATTILA
}

\begin{abstract}
We study subsets $E$ of $\mathbf{R}^{n}$ which are $H^{s}$ measurable and have $0<H^{s}(E)<\infty$, where $H^{s}$ is the s-dimensional Hausdorff measure. Given an integer $k, s \leq k \leq n$, we consider $\operatorname{six}(s, k)$ regularity definitions for $E$ in terms of $k$-dimensional subspaces or surfaces of $\mathbf{R}^{n}$. If $s=k$, they all agree with the $\left(H^{k}, k\right)$ rectifiability in the sense of Federer, but in the case $s<k$ we show that only two of them are equivalent. We also study sets with positive lower density, and projection properties in connection with these regularity definitions.
\end{abstract}

1. Introduction. Let $E$ be an $s$-set, that is, a subset of the Euclidean $n$-space $\mathbf{R}^{n}$ which is measurable with respect to the $s$-dimensional Hausdorff measure $H^{s}$ and for which $0<H^{s}(E)<\infty$. Here $s$ is a real number, $0<s<n$. If $s=k$ is an integer, then according to the theories of Besicovitch and Federer (see [FK and $\mathbf{F H}$ ),$E$ can be split into two parts, regular and irregular (or in Federer's terminology $\left(H^{k}, k\right)$ rectifiable and purely $\left(H^{k}, k\right)$ unrectifiable), the regular part having similar geometric measure theoretic properties as nice $k$-dimensional surfaces whereas the irregular part has completely opposite behavior. It is a fractal in the sense of Mandelbrot [MB]. Moreover, several such properties characterize regularity and irregularity. Among these properties, many refer to relations between the $s$-set $E$ and $k$-planes, such as existence of tangent $k$-planes, orthogonal projections into linear $k$-planes and covering $E$ with countably many Lipschitz or $C^{1}$ images of (the $k$-plane) $\mathbf{R}^{k}$. For example, an irregular $k$-set projects on almost all $k$-planes into a set of $k$-dimensional measure zero but the projections of a regular $k$-set have generically positive $k$-measure.

In this paper we study the question whether an analogous theory could be developed in the case $s<k$. The possibility of such a theory was inquired by Brian White in [W] where he more specifically asked if, when $s<k$, there exists an $s$-set $E$ whose projections on $k$-planes have $s$-dimensional measure zero. Such a set could be considered more irregular than e.g. an $s$-set lying on a $k$-plane, which clearly has almost all projections of positive $s$-measure. One could then ask if such an irregular projection behavior is connected with some other irregular behavior. It turns out that such sets do exist and that there are some relations between different types of $k$-dimensional regularity and irregularity of $s$-sets.

We shall study the following $\operatorname{six}(s, k)$ regularity definitions for an $s$-set $E, s \leq k$. If $s=k$, they all agree with Besicovitch-Federer regularity. But, for example, a

Received by the editors October 30, 1986.

1980 Mathematics Subject Classification (1985 Revision). Primary 28A75.

Key words and phrases. $(s, k)$ regular sets, Hausdorff measures, tangent planes, orthogonal projections. 
purely $\left(H^{1}, 1\right)$ unrectifiable subset of $\mathbf{R}^{3}$ may fall into any (or none) of these $(1,2)$ regularity classes. For more precise definitions, see $\S 3$.

(1) $C^{1}$ regularity: $H^{s}$ almost all of $E$ can be covered with countably many $k$ dimensional $C^{1}$ submanifolds of $\mathbf{R}^{n}$.

(2) Lipschitz regularity: $H^{s}$ almost all of $E$ can be covered with countably many Lipschitz images of $\mathbf{R}^{k}$.

(3) Tangential regularity: $H^{s}$ almost all of $E$ can be split into countably many subsets $E_{i}$ such that at every point $a$ of $E_{i}$ the tangent vectors of $E_{i}$ at $a$ lie on a $k$-plane.

(4) Conical regularity: $H^{s}$ almost all of $E$ can be split into countably many subsets $E_{i}$ such that every point of $E_{i}$ is a vertex of an open cone $C$ around an $(n-k)$-plane with $C \cap E_{i}=\varnothing$.

(5) Approximate tangential regularity: At $H^{s}$ almost every point $a$ of $E$ the $H^{s}$ approximate tangent vectors of $E$ at $a$ lie on a $k$-plane.

(6) Approximate conical regularity: $H^{s}$ almost every point $a$ of $E$ is a vertex of an open cone $C$ around an $(n-k)$-plane such that the $s$-dimensional density of $C \cap E$ at $a$ is zero.

For each item (1)-(6) one can define the corresponding irregularity of $E$ by requiring that $E$ contains no regular subset of positive $H^{s}$ measure.

We shall see that (1) $\Leftrightarrow(3) \Rightarrow(4) \Rightarrow(2),(3) \Rightarrow(5) \Rightarrow(6),(4) \Rightarrow(6)$, and that the other implications, except those logically following from the above, are false. So we have five different definitions for $(s, k)$ regularity. In $\S 4$ we consider sets $E$ with positive lower density;

(7) $\liminf _{r \downarrow 0} r^{-s} H^{s}\{x \in E:|x-a| \leq r\}>0$ for $H^{s}$ almost all $a \in E$.

We prove that under this condition (2) always holds, $(3) \Leftrightarrow(5),(4) \Leftrightarrow(6)$, and that the other possible implications are false. Thus assuming (7) we are left with only two different genuine regularity concepts; tangential and conical.

In $\S 5$ we consider the consequences these different kinds of $(s, k)$ regularity bear on projection properties. We shall show that (1) implies that the projection of $E$ on almost every linear $k$-plane has positive $H^{s}$ measure, and (4) implies that the set of such $k$-planes has positive measure, but also its complement may have positive measure. We shall also construct examples to show that (2), (5), and (6) imply nothing on the $H^{s}$ measures of the projections on $k$-planes.

Compared to the completeness and depth of the Besicovitch-Federer structure theory, our theory is merely a tentative first step, and it remains to be seen if it is possible to achieve deeper theorems. For projections this would mean exploring the projection properties of the various $(s, k)$ irregular sets, which is likely to be a much more difficult question than establishing the projection properties of the $(s, k)$ regular sets given above.

Another aspect which seems very difficult is to find the relations between the $(s, k)$ regularity definitions and the behavior of the density ratios. Even in the case $s=k$ there are still substantial open problems (although outstanding progress was made recently by David Preiss who in $[\mathbf{P}]$ verified among other things the old conjecture of Federer that $k$ regularity is equivalent to the existence of the limit, when $s=k$,

$$
\lim _{r \downarrow 0} r^{-s} H^{s}\{x \in E:|x-a| \leq r\}
$$


for $H^{s}$ almost all $a \in E$ ). If $s$ is not an integer, Marstrand [MJ] has shown that this limit fails to exist for $H^{s}$ almost all $a \in E$. But do the above $(s, k)$ regularity properties force some restrictions on the behavior of $r^{-s} H^{s}\{x \in E:|x-a| \leq r\}$ that are not present for all $s$-sets $E$ ?

Part of the results of this paper were included in the thesis [MM].

2. Preliminaries. Throughout this paper $k$ and $n$ will be integers with $0<$ $k<n$. For $0 \leq s \leq n$ we define $s$-dimensional Hausdorff measure $H^{s}$ as

$$
H^{s}(E)=\lim _{\delta \downarrow 0} \inf \left\{\sum d\left(S_{i}\right)^{s}: E \subset \bigcup_{i=1}^{\infty} S_{i}, d\left(S_{i}\right) \leq \delta\right\}, \quad E \subset \mathbf{R}^{n},
$$

where $d$ stands for the diameter. Then $H^{n}$ is a constant multiple of the Lebesgue measure $L^{n}$. By an $s$-set we mean an $H^{s}$ measurable set $E$ with $0<H^{s}(E)<\infty$. We let $B(a, r)$ denote the closed ball

$$
B(a, r)=\left\{x \in \mathbf{R}^{n}:|x-a| \leq r\right\}, \quad a \in \mathbf{R}^{n}, 0<r<\infty,
$$

and define the $s$-dimensional upper and lower densities of a set $E \subset \mathbf{R}^{n}$ at a point $a \in \mathbf{R}^{n}$ by

$$
\begin{aligned}
& \theta^{* s}(E, a)=\limsup _{r \downarrow 0}(2 r)^{-s} H^{s}(E \cap B(a, r)), \\
& \theta_{*}^{s}(E, a)=\liminf _{r \downarrow 0}(2 r)^{-s} H^{s}(E \cap B(a, r)) .
\end{aligned}
$$

The following basic density theorem will be very useful for us (for a proof see [FK, Chapter 2 or FH, 2.10.19]):

2.1 THEOREM. Suppose $E \subset \mathbf{R}^{n}$ with $H^{s}(E)<\infty$.

(1) $2^{-s} \leq \theta^{* s}(E, a) \leq 1$ for $H^{s}$ almost all $a \in E$.

(2) If $E$ is $H^{s}$ measurable, $\theta^{* s}(E, a)=0$ for $H^{s}$ almost all $a \in \mathbf{R}^{n} \backslash E$.

(3) If $F \subset E$ are $H^{s}$ measurable, $\theta^{* s}(F, a)=\theta^{* s}(E, a)$ for $H^{s}$ almost all $a \in F$.

The Grassmannian manifold of $k$-dimensional linear subspaces of $\mathbf{R}^{n}$ is denoted by $G(n, k)$. The orthogonal complement of $V \in G(n, k)$ is $V^{\perp} \in G(n, n-k)$, and $P_{V}: \mathbf{R}^{n} \rightarrow V$ and $Q_{V}: \mathbf{R}^{n} \rightarrow V^{\perp}$ denote the orthogonal projections. With the metric $d$,

$$
d(V, W)=\left\|P_{V}-P_{W}\right\|,
$$

$G(n, k)$ is a compact metric space. Here $\|L\|=\sup _{|x|=1}|L x|$ is the usual norm of the linear map $L$. There is a unique orthogonally invariant Radon probability measure $\mathcal{V}_{n, k}$ on $G(n, k)$. If $n=2, k=1$, we shall identify a line $L \in G(2,1)$ with the angle $\theta \in[0, \pi)$ which it makes with the positive $x$-axis. Then $\mathcal{V}_{2,1}$ is just the normalized Lebesgue measure on $[0, \pi)$. Analogous identifications lead to interpretations of $\nu_{n, 1}$ and $\nu_{n, n-1}$ as the normalized area measure on the unit sphere $\{x:|x|=1\}$. Frequently we shall consider the cones

$$
\begin{aligned}
X(a, r, V, t) & =\{x: \operatorname{dist}(x-a, V)<t|x-a|,|x-a| \leq r\} \\
& =\left\{x:\left|Q_{V}(x-a)\right|<t|x-a|,|x-a| \leq r\right\}
\end{aligned}
$$

defined for $a \in \mathbf{R}^{n}, V \in G(n, k), 0<r \leq \infty$, and $0<t<1$. Note that if $n=2$ and $k=1$, this is the two-sided angular sector with vertex $a$, central axis $V+a$, and 
opening angle $2 \overline{\operatorname{arc}} \sin t$. The following identity and inclusion are easy to verify for $a \in \mathbf{R}^{n}, 0<r \leq \infty, 0<t, u<1$, and $V, W \in G(n, k)$ :

$$
\begin{aligned}
& X\left(a, r, V^{\perp},\left(1-t^{2}\right)^{1 / 2}\right)=B(a, r) \backslash(\text { Closure } X(a, r, V, t)), \\
& \text { if }\left\|P_{V}-P_{W}\right\|<u-t \text {, then } X(a, r, V, t) \subset X(a, r, W, u) .
\end{aligned}
$$

3. Definitions for $(s, k)$ regularity. In all the definitions below $0<s \leq k \leq n$ and $E$ is an $H^{s}$ measurable subset of $\mathbf{R}^{n}$ with $H^{s}(E)<\infty$.

3.1 DEFINITION. $E$ is $(s, k)$ Lipschitz regular, if there are Lipschitz maps $f_{i}: \mathbf{R}^{k}$ $\rightarrow \mathbf{R}^{n}$ such that

$$
H^{s}\left(E \backslash \bigcup_{i=1}^{\infty} f_{i}\left(\mathbf{R}^{k}\right)\right)=0
$$

Note. It would be equivalent to assume that the $f_{i}$ 's are defined on some subsets of $\mathbf{R}^{k}$ since Lipschitz maps can always be extended [FH, 2.10.43].

3.2 DEFINITION. $E$ is $(s, k) C^{1}$ regular if there are $k$-dimensional $C^{1}$ submanifolds $M_{i}$ of $\mathbf{R}^{n}$ such that

$$
H^{s}\left(E \backslash \bigcup_{i=1}^{\infty} M_{i}\right)=0 .
$$

3.3 DEFINITION. $E$ is $(s, k)$ tangentially regular if there are $H^{s}$ measurable sets $E_{0}, E_{1}, E_{2}, \ldots$ such that

$$
E=\bigcup_{i=0}^{\infty} E_{i} \quad \text { with } H^{s}\left(E_{0}\right)=0
$$

and for all $a \in E_{i}, i=1,2, \ldots$, there is $V \in G(n, k)$ such that for every $t, 0<t<1$, there is $r>0$ for which

$$
E_{i} \cap X\left(a, r, V^{\perp}, t\right)=\varnothing .
$$

$V$ is then called a tangent $k$-plane of $E_{i}$ at a.

3.4 DEFINITION. $E$ is $(s, k)$ conically regular if there are $H^{s}$ measurable sets $E_{0}, E_{1}, E_{2}, \ldots$ such that

$$
E=\bigcup_{i=0}^{\infty} E_{i} \quad \text { with } H^{s}\left(E_{0}\right)=0,
$$

and for all $a \in E_{i}, i=1,2, \ldots$, there are $r>0, V \in G(n, k)$ and $0<t<1$ such that

$$
E_{i} \cap X\left(a, r, V^{\perp}, t\right)=\varnothing .
$$

Note. By further subdividing each $E_{i}$ it is not difficult to see that we can actually take $r, V$, and $t$ independent of $a \in E_{i}$. Clearly we can also choose $E_{i}$ 's to be disjoint. Observe that in a sense 3.3 is a limiting case of 3.4 when $t \rightarrow 1$.

3.5 DEFINITION. $E$ is $(s, k)$ approximately tangentially regular if for $H^{s}$ almost all $a \in E$ there is $V \in G(n, k)$ such that for all $0<t<1$

$$
\lim _{r \downarrow 0} r^{-s} H^{s}\left(E \cap X\left(a, r, V^{\perp}, t\right)\right)=0 .
$$

$V$ is then called an approximate tangent $k$-plane of $E$ at $a$. 
3.6 DEFINITION. $E$ is $(s, k)$ approximately conically regular if for $H^{s}$ almost all $a \in E$ there are $V \in G(n, k)$ and $0<t<1$ such that

$$
\lim _{r \downarrow 0} r^{-s} H^{s}\left(E \cap X\left(a, r, V^{\perp}, t\right)\right)=0 .
$$

3.7 REMARKS. (1) If $E=\bigcup_{i=0}^{\infty} E_{i}$ as in 3.3, then by $2.1(2) \theta^{* s}\left(E \backslash E_{i}, a\right)=0$ for $H^{s}$ almost all $a \in E_{i}$. If at such a point $a$, a tangent $k$-plane of $E_{i}$ exists, it is also an approximate tangent $k$-plane of $E$.

(2) It is possible that $H^{s}(E)>0$ and $E$ has several tangent $k$-planes at all of its points. For example, take $s=1, k=2, n=3$ and $E$ a line segment. All the 2-planes containing $E$ are its tangent 2-planes. But this is a bit artificial as $E$ has a unique tangent 1-plane. In fact, whenever a set $E$ has two distinct (approximate) tangent $k$-planes $V$ and $W$ at a point $a$, then also $V \cap W$ is an (approximate) tangent plane for $E$ at $a$. (If $V \cap W=\{0\}$, this means that $a$ is an isolated point of $E$ or, in the approximate case, that $\theta^{* s}(E, a)=0$.) If follows that if $E$ has an (approximate) tangent plane at $a$, it has a unique (approximate) tangent plane at $a$ of the smallest possible dimension. At $H^{s}$ almost all points of $E$ this minimal dimension is at least $s$ due to the upper density properties of $s$-sets (see [S]).

(3) For $a \in \mathbf{R}^{n}, 0<r<\infty, V \in G(n, k)$ and $0<\delta<1$, let $S(a, r, V, \delta)=$ $\{x: \operatorname{dist}(x-a, V) \leq \delta r,|x-a| \leq r\}$. Then, if $0<t<1$,

$$
\begin{gathered}
X(a, r, V, t) \subset S(a, r, V, t), \\
S(a, r, V, \delta) \subset X(a, r, V, t) \cup B(a, \delta r / t) .
\end{gathered}
$$

Complementing these inclusions, one deduces that if $\theta^{* s}(E, a)<\infty$, then $V$ is an approximate tangent $k$-plane of $E$ at $a$ if and only if for all $\delta>0$

$$
\lim _{r \downarrow 0} r^{-s} H^{s}(E \cap(B(a, r) \backslash S(a, r, V, \delta)))=0 .
$$

Recall from 2.1(1) that $\theta^{* s}(E, a) \leq 1$ for $H^{s}$ almost all $a \in E$.

(4) Let $P$ denote any of the properties $C^{1}$, Lipschitz, etc. occurring in Definitions 3.1-3.6. It is obvious that $(s, k) P$ regularity implies $(s, k+1) P$ regularity. One is then led to define the $P$ degree of regularity of an $s$-set $E$ as the smallest integer $k$ such that $E$ is $(s, k) P$ regular. We agree that all $s$-sets in $\mathbf{R}^{n}$ are $(s, n) \mathcal{P}$ regular. Let us say that $E$ is properly $(s, k) P$ regular if $E$ is $(s, k) P$ regular and the $P$ degree of regularity of every $s$-subset of $E$ equals $k$. We leave it as an exercise to show that every $s$-set $E \subset \mathbf{R}^{n}$ admits a decomposition $E=\bigcup_{i=k}^{n} E_{i}$, where each $E_{i}$ is properly $(s, i) P$ regular and $k$ is the smallest integer with $s \leq k$.

(5) Let $P$ be as in the previous remark. We say that an $s$-set $E$ is $(s, k) P$ irregular if $E$ has no $(s, k) P$ regular $s$-subsets; that is, $H^{s}(E \cap F)=0$ for every $(s, k) P$ regular $s$-set $F$. Any $s$-set $E$ can be decomposed as $E=A \cup B$ where $A$ is $(s, k) P$ regular and $B$ is $(s, k) P$ irregular. Indeed, if $E$ is not $(s, k) P$ irregular, select for every $i=1,2, \ldots$ an $(s, k) P$ regular subset $E_{i}$ of $E$ with

$$
\sup \left\{H^{s}(F): F \subset E(s, k) P \text { regular }\right\} \leq H^{s}\left(E_{i}\right)+1 / i,
$$

and take $A=\bigcup_{i=1}^{\infty} E_{i}$. This simple argument is from [R].

Analogously to (4) one can define the $P$ degree of irregularity, properly $(s, k) P$ irregular sets, and obtain the corresponding decomposition. 
In each case there is an alternate definition for $(s, k) P$ irregularity not referring to $(s, k) \mathcal{P}$ regularity. We omit their rather straightforward formulations and proofs and only state two cases:

$E$ is $(s, k) C^{1}$ irregular if and only if $H^{s}(E \cap M)=0$ for every $k$-dimensional $C^{1}$ submanifold $M$ of $\mathbf{R}^{n}$.

$E$ is $(s, k)$ approximately tangentially irregular if and only if for $H^{s}$ almost all $a \in E$ and for all $V \in G(n, k)$, there is $t, 0<t<1$, such that

$$
\limsup _{r \downarrow 0} r^{-s} H^{s}\left(E \cap X\left(a, r, V^{\perp}, t\right)\right)>0 .
$$

The conical $(s, k)$ irregularity can be defined in terms of Lipschitz graphs (see $3.10)$.

In the following theorem we give all the valid implications between the different definitions of $(s, k)$ regularity (except those logically following from the stated ones). Later on we shall present examples to show that the remaining implications are false. In the proof of Theorem 3.9 we shall need the following measurability lemma.

3.8 LEMMA. Suppose that a Borel set $E \subset \mathbf{R}^{n}$ has a tangent $k$-plane at all of its points. Then there is a Borel function $g: E \rightarrow G(n, k)$ such that $g(a)$ is a tangent $k$-plane for $E$ at $a$.

Proof. For $a, x_{1}, \ldots, x_{k} \in \mathbf{R}^{n}$ with $\left|x_{i}-a\right|>0$, let

$$
J\left(a, x_{1}, \ldots, x_{k}\right)=\frac{x_{1}-a}{\left|x_{1}-a\right|} \wedge \cdots \wedge \frac{x_{k}-a}{\left|x_{k}-a\right|} \in \Lambda_{k} \mathbf{R}^{n} .
$$

(For the notation and definitions on multilinear algebra see Chapter 1 of $[\mathbf{F H}]$. ) Recall that the geometric interpretation of $V_{1} \wedge \cdots \wedge V_{m}$ is the parallelepiped generated by the vectors $V_{1}, \ldots, V_{k}$. The norm $\left|V_{1} \wedge \cdots \wedge V_{k}\right|$ gives its $k$-dimensional volume.

For $\delta>0$ define

$$
E_{\delta}=\bigcap_{j=1}^{\infty}\left\{a \in E: \sup \left\{\left|J\left(a, x_{1}, \ldots, x_{k}\right)\right|: x_{i} \in E, 0<\left|x_{i}-a\right|<1 / j\right\}>\delta\right\} .
$$

Then $E_{\delta}$ is a countable intersection of relatively open subsets of $E$, whence it is a Borel set. For each positive integer $m$ express $\Lambda_{k} \mathbf{R}^{n}$ as

$$
\Lambda_{k} \mathbf{R}^{n}=\bigcup_{i=1}^{p_{m}} \Lambda_{m, i} \quad \text { with } d\left(\Lambda_{m, i}\right)<1 / m
$$

where the $\Lambda_{m, i}$ 's are open. Let

$$
\begin{aligned}
E_{\delta, m, i}^{\prime}=\left\{a \in E_{\delta}: \sup \{\mid\right. & J\left(a, x_{1}, \ldots, x_{k}\right) \mid: x_{l} \in E, \\
& \left.\left.0<\left|x_{l}-a\right|<1 / m, J\left(a, x_{1}, \ldots, x_{k}\right) \in \Lambda_{m, i}\right\}>\delta\right\} .
\end{aligned}
$$

Then $E_{\delta}=\bigcup_{i} E_{\delta, m, i}^{\prime}$, and each $E_{\delta, m, i}^{\prime}$ is a Borel set as a relatively open subset of $E_{\delta}$. Select disjoint Borel sets $E_{\delta, m, i} \subset E_{\delta, m, i}^{\prime}$ such that $E_{\delta}=\bigcup_{i} E_{\delta, m, i}$. Define Borel functions

$$
T_{\delta, m}: E_{\delta} \rightarrow \Lambda_{k} \mathbf{R}^{n}
$$

such that $T_{\delta, m} \mid E_{\delta, m, i}$ is a constant $J_{\delta, m, i} \in \Lambda_{m, i}$ for every $i$ with $E_{\delta, m, i} \neq \varnothing$. Let $V_{\delta, m}(a) \in G(n, k)$ be the subspace of $\mathbf{R}^{n}$ associated with $T_{\delta, m}(a)$ (see [FH, 
1.6.1]). Let $a \in E_{\delta}$. Then there are $x_{m, 1}, \ldots, x_{m, k} \in E, m=1,2, \ldots$, such that $0<\left|x_{m, i}-a\right|<1 / m$,

$$
\left|J\left(a, x_{m, 1}, \ldots, x_{m, k}\right)\right|>\delta
$$

and

$$
\left|J\left(a, x_{m, 1}, \ldots, x_{m, k}\right)-T_{\delta, m}(a)\right|<2 / m \text {. }
$$

Since $E$ has a tangent $k$-plane $V_{a}$ at $a$, the $k$-planes spanned by $x_{m, 1}-a, \ldots, x_{m, k}-a$ converge to $V_{a}$ as $m \rightarrow \infty$ because of (1), and (2) implies that also $V_{\delta, m}(a) \rightarrow V_{a}$. Denote

$$
g_{\delta}(a)=\lim _{m \rightarrow \infty} V_{\delta, m}(a) \text { for } a \in E_{\delta}
$$

Then $g_{\delta}$ is a Borel function.

Let $E^{j}, j=1, \ldots, k$, be the set of those $a \in E$ where $E$ has a unique tangent $j$-plane $V_{a}$ (and thus no tangent $(j-1)$-plane). Then $E^{k}=\bigcup_{p=1}^{\infty} E_{1 / p}$. Hence $E^{k}$ is a Borel set and we can define

$$
g^{k}(a)=g_{1 / p}(a)=V_{a} \quad \text { for } a \in E_{1 / p} .
$$

Dealing with $E^{k-1}, \ldots, E^{1}$ as above with $E^{k}$, we find Borel functions

$$
g^{j}: E^{j} \rightarrow G(n, j) \quad \text { with } g^{j}(a)=V_{a} .
$$

It is not difficult to find Borel functions $h^{j}: E^{j} \rightarrow G(n, k-j), j=1, \ldots, k$, with $g^{j}(a) \cap h^{j}(a)=\{0\}$. Since $E=\bigcup_{j=1}^{k} E^{j}$, the required $g$ can be defined by

$$
g(a)=g^{j}(a) \oplus h^{j}(a) \text { for } a \in E^{j} .
$$

3.9 THEOREM. Suppose $E \subset \mathbf{R}^{n}$ is $H^{s}$ measurable with $H^{s}(E)<\infty$, and $s \leq k$. Then

(1) $E$ is $(s, k) C^{1}$ regular if and only if it is $(s, k)$ tangentially regular.

(2) If $E$ is $(s, k) C^{1}$ regular, it is $(s, k)$ Lipschitz regular.

(3) If $E$ is $(s, k)$ tangentially regular, it is $(s, k)$ conically regular.

(4) If $E$ is $(s, k)$ conically regular, it is $(s, k)$ Lipschitz regular.

(5) If $E$ is $(s, k)$ approximately tangentially regular, it is $(s, k)$ approximately conically regular.

(6) If $E$ is $(s, k)$ tangentially regular, it is $(s, k)$ approximately tangentially regular.

(7) If $E$ is $(s, k)$ conically regular, it is $(s, k)$ approximately conically regular.

PROOF. (2), (3), and (5) are obvious. (6) follows from Remark 3.7(1), and (7) is a consequence of a similar argument.

PROOF OF (4) (ESSENTIALLY FROM [FH, 3.3.5]). It suffices to show (cf. the note following 3.4) that if $V \in G(n, k), 0<r<\infty, 0<t<1, d(E)<r$, and $E \cap X\left(a, r, V^{\perp}, t\right)=\varnothing$ for $a \in E$, then $E$ is a Lipschitz image of a subset of $V$. If $a \in E, b \in \mathbf{R}^{n},\left|P_{V} a-P_{V} b\right|<t|a-b|$, and $|a-b| \leq r$, then $b \in X\left(a, r, V^{\perp}, t\right)$ (since $Q_{V \perp}=P_{V}$ ) and so $b \notin E$. Thus $\left|P_{V} a-P_{V} b\right| \geq t|a-b|$ for $a, b \in E$, whence $P_{V} \mid E$ has a Lipschitz inverse. But $E=\left(P_{V} \mid E\right)^{-1}\left(P_{V} E\right)$, and (4) follows.

PROOF OF (1). If $E$ is $(s, k) C^{1}$ regular, we can write $E$ as $\bigcup_{i=0}^{\infty} E_{i}$ where $H^{s}\left(E_{0}\right)=0$ and $E_{i}$ is a subset of a $k$-dimensional $C^{1}$ submanifold $M_{i}$ for 
$i=1,2, \ldots$ If $a \in E_{i}, M_{i}$ has a tangent $k$-plane at $a$, which is also a tangent $k$-plane of $E_{i}$. Hence $E$ is $(s, k)$ tangentially regular.

Assume $E$ is $(s, k)$ tangentially regular. We have to split $E$ into $E_{0}, E_{1}, E_{2}, \ldots$ such that $H^{s}\left(E_{0}\right)=0$ and $E_{i}$ is contained in a $k$-dimensional $C^{1}$ submanifold for $i=1,2, \ldots$. By performing several partitionings we shall reduce the problem to subcases where $E$ satisfies some extra assumption. First using Lemma 3.8 and the fact that $E$ is a union of countably many compact sets and a set of $H^{s}$ measure zero, we may assume that $E$ is compact and has at all of its points a tangent $k$-plane $V_{a}$ such that the map $a \rightarrow V_{a}$ is Borel measurable. By dividing $E$ further we may assume that

$$
\left|P_{V_{a}}(a-b)\right| \geq \frac{3}{4}|a-b| \text { for } a, b \in E,
$$

and that there is $W \in G(n, k)$ such that

$$
\left\|P_{V_{a}}-P_{W}\right\| \leq \frac{1}{2} \quad \text { for } a \in E .
$$

Then for $a, b \in E$

$$
\left|P_{W} a-P_{W} b\right| \geq\left|P_{V_{a}}(a-b)\right|-\left\|P_{V_{a}}-P_{W}\right\||a-b| \geq|a-b| / 4 .
$$

Hence $P_{W} \mid E$ is one-to-one with Lipschitz inverse $f$,

$$
f=\left(P_{W} \mid E\right)^{-1}: A \rightarrow E \quad \text { with } A=P_{W} E .
$$

Due to (8) $P_{W} \mid V_{a}$ is injective for all $a \in E$. Let

$$
L_{x}=\left(P_{W} \mid V_{f(x)}\right)^{-1}: W \rightarrow V_{f(x)} \text { for } x \in A .
$$

The map $x \rightarrow L_{x}$ is Borel measurable and therefore uniformly continuous in a compact subset $A_{0}$ of $A$ with $H^{s}\left(f\left(A \backslash A_{0}\right)\right)$ arbitrarily small. We apply Lusin's theorem to the measure $B \rightarrow H^{s}(f B), B \subset E$. We may thus assume that $x \rightarrow L_{x}$ is uniformly continuous in $A$; that is,

$$
\limsup _{\delta \downarrow 0}\left\{\left\|L_{x}-L_{y}\right\|: x, y \in A,|x-y| \leq \delta\right\}=0 .
$$

For $j=1,2, \ldots$ and $a \in E$, define

$$
f_{j}(a)=\sup \left\{\operatorname{dist}\left(a-b, V_{a}\right) /|a-b|: b \in E, 0<|a-b| \leq 1 / j\right\} .
$$

Then that $V_{a}$ is a tangent plane for $E$ at $a$ means exactly that $f_{j}(a) \rightarrow 0$ as $j \rightarrow \infty$. By Egoroff's theorem the convergence is uniform outside a relatively open subset of $E$ of arbitrarily small measure, and we may again assume that $f_{j} \rightarrow 0$ uniformly on $E$. This gives

$$
\lim _{\delta \downarrow 0} \sup \left\{\operatorname{dist}\left(f(x)-f(y), V_{f(x)}\right) /|x-y|: x, y \in A, 0<|x-y| \leq \delta\right\}=0 .
$$

For $x \in A$ define an affine map $P_{x}: W \rightarrow \mathbf{R}^{n}$ by

$$
P_{x}(y)=L_{x}(y-x)+f(x) .
$$

Then $P_{x}(x)=f(x), D P_{x}(y)=L_{x}$ for $y \in W$. Since $P_{W}\left(f(x)-f(y)-L_{x}(x-y)\right)=0$, we have by (8)

$$
\begin{aligned}
& \left|P_{V_{f(x)}}\left(f(x)-f(y)-L_{x}(x-y)\right)\right| \\
& \quad \leq\left\|P_{V_{f(x)}}-P_{W}\right\|\left|f(x)-f(y)-L_{x}(x-y)\right| \leq \frac{1}{2}\left|f(x)-f(y)-L_{x}(x-y)\right|
\end{aligned}
$$


whence

$$
\begin{aligned}
\operatorname{dist}\left(f(x)-f(y), V_{f(x)}\right) & =\left|Q_{V_{f(x)}}\left(f(x)-f(y)-L_{x}(x-y)\right)\right| \\
& \geq \frac{1}{2}\left|f(x)-f(y)-L_{x}(x-y)\right| .
\end{aligned}
$$

Since $P_{x}(y)-P_{y}(y)=f(x)-f(y)-L_{x}(x-y)$, we infer using (10)

$$
\lim _{\delta \downarrow 0} \sup \left\{\left|P_{x}(y)-P_{y}(y)\right| /|x-y|: x, y \in A, 0<|x-y| \leq \delta\right\}=0 .
$$

Applying (9), (11), and Whitney's extension theorem (see e.g. [FH, 3.1.14]), we find a $C^{1}$ extension $g: W \rightarrow \mathbf{R}^{n}$ of $f$. Then also $h, h(x)=g(x)+x-P_{W}(g(x))$, $x \in W$, is a $C^{1}$ extension of $f$. Since $P_{W}(h(x))=x$ for $x \in W$, the image of $h$ is a $C^{1}$ submanifold containing $E$.

3.10 Lipschitz graphs. We say that a subset $L$ of $\mathbf{R}^{n}$ is a Lipschitz $k$-graph if there are $V \in G(n, k), B \subset V$, and a Lipschitz map $f: B \rightarrow V^{\perp}$ such that $L=$ $\{x+f(x): x \in B\}$. If $f$ is $K$-Lipschitz, then for $a=x+f(x)$ and $b=y+f(y) \in L$,

$$
|a-b|=\left(|x-y|^{2}+|f(x)-f(y)|^{2}\right)^{1 / 2} \leq\left(1+K^{2}\right)^{1 / 2}\left|P_{V} a-P_{V} b\right|,
$$

whence $L \cap X\left(a, \infty, V^{\perp}, t\right)=\varnothing$ with $t=\left(1+K^{2}\right)^{-1 / 2}$. Using this and the proof of Theorem 3.9(4), we obtain the following characterization of conical regularity in terms of Lipschitz graphs.

3.11 THEOREM. Let $E$ be an $H^{s}$ measurable subset of $\mathbf{R}^{n}$ with $H^{s}(E)<\infty$, $s \leq k$. Then $E$ is $(s, k)$ conically regular if and only if there are Lipschitz $k$-graphs $L_{1}, L_{2}, \ldots$ such that

$$
H^{s}\left(E \backslash \bigcup_{i=1}^{\infty} L_{i}\right)=0 .
$$

We shall now give examples to disprove the implications not contained in Theorem 3.8. For simplicity we shall perform our constructions only in the plane, but similar ideas can also be used in general dimensions.

In some cases we shall postpone the constructions and arguments to later sections where they will also be used for other purposes.

3.12 Lipschitz regularity does not imply approximate conical regularity; hence Lipschitz regularity does not imply any of the other regularity concepts.

PROOF. See 5.3.

3.13 Conical regularity does not imply approximate tangential regularity.

PROOF. Use the set of 5.4.

3.14. Approximate tangential regularity does not imply Lipschitz regularity.

PROOF. Let $0<s<1$. We shall construct a Cantor-type set starting from a closed square $Q_{1,1}$ with sides parallel to the coordinate axis.

Suppose the squares $Q_{k, 1}, \ldots, Q_{k, p_{k}}$ have been selected, each having side-length $d_{k}$. Let $Q$ be one of them with center $\mathbf{a} \equiv(a, b)$. Choose an integer $n_{k}$ such that $n_{k} d_{k}>k$ and partition $\left\{(x, y) \in Q:|y-b| \leq d_{k} /\left(2 n_{k}\right)\right\}$ into $n_{k}^{3}$ squares $P_{i}^{\prime}$ of the same size (see Figure 1). Define $d_{k+1}$ by $n_{k}^{3} d_{k+1}^{s}=d_{k}^{s}$, and for each $i$ let $P_{i}$ be the closed square with the same center as $P_{i}^{\prime}$ and with side-length $d_{k+1}$. Let $Q_{k+1, j}$, $j=1, \ldots, p_{k+1}$, be all such squares $P_{i}$ constructed inside the squares $Q_{k, j}$. Define a compact set $E$ by

$$
E=\bigcap_{k=1}^{\infty} \bigcup_{j=1}^{p_{k}} Q_{k, j} .
$$




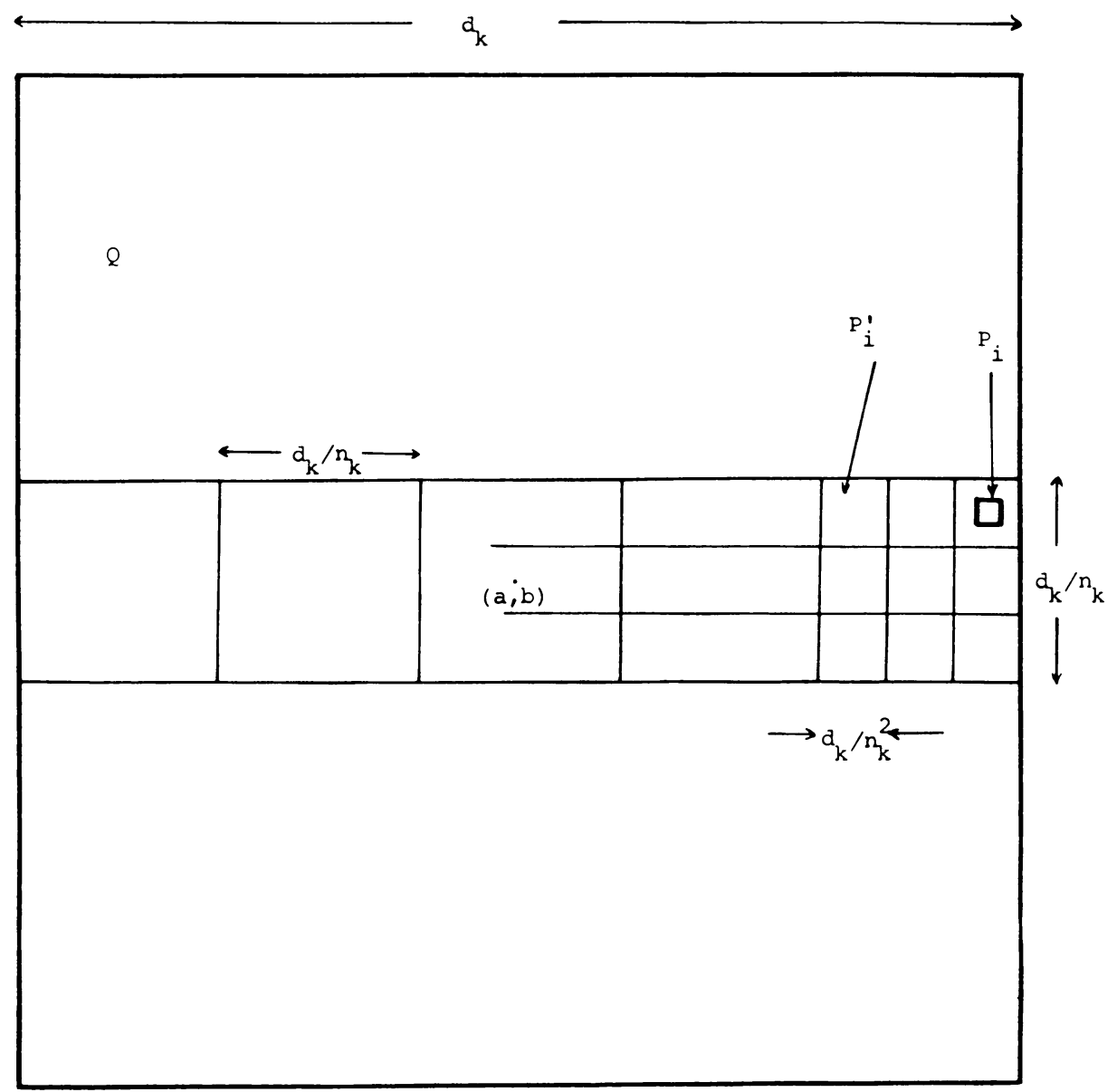

FIGURE 1

Then by standard arguments $0<H^{s}(E)<\infty$ and

$$
H^{s}\left(E \cap Q_{k, j}\right) \leq\left(2 d_{k}\right)^{s} \text { for all } k, j .
$$

We shall first show that the $x$-axis, $L=\{(x, y): y=0\}$, is an approximate tangent line for all points of $E$. Let $\mathbf{a} \equiv(a, b) \in E, \varepsilon>0$, and $0<\delta<1$. As $\theta^{* s}(E,(a, b))<\infty$, we can use the alternate definition of 3.7(3) for tangent planes. Let $0<r<d_{1}$ and choose $k$ such that $d_{k+1} \leq r<d_{k}$. If $\delta r \geq d_{k} / n_{k}$, then

$$
B(\mathbf{a}, x) \cap\{(x, y):|y-b|>\delta r\} \cap E=\varnothing .
$$

Suppose $\delta r<d_{k} / n_{k}$. If $r>d_{k} /\left(2 n_{k}\right), B(\mathbf{a}, r)$ contains no more than $2 n_{k}^{3} r / d_{k}$ squares $Q_{k+1, j}$ (estimate the number of such squares in a rectangle with sidelengths $2 r$ and $\left.d_{k} / n_{k}\right)$. Hence

$$
\begin{aligned}
H^{s}(E \cap B(\mathbf{a}, r)) & \leq 2 n_{k}^{3} r d_{k}^{-1}\left(2 d_{k+1}\right)^{s}=2^{s+1} r d_{k}^{s-1} \\
& <2^{s+1} r\left(\delta r n_{k}\right)^{s-1}=2^{s+1}\left(\delta n_{k}\right)^{s-1} r^{s}<\varepsilon r^{s}
\end{aligned}
$$

for sufficiently large $k$. If $r \leq d_{k} /\left(2 n_{k}\right), B(\mathbf{a}, r)$ contains no more than $4 n_{k}^{4} r^{2} / d_{k}^{2}$ squares $Q_{k+1, j}$ (estimate the number of such squares in a square with side-length 
$2 r)$. Hence

$$
\begin{aligned}
H^{s}(E \cap B(\mathbf{a}, r)) & \leq 4 n_{k}^{4} r^{2} d_{k}^{-2}\left(2 d_{k+1}\right)^{s}=2^{s+2} n_{k} r^{2} d_{k}^{s-2} \\
& <2^{s+2} n_{k} r^{2}\left(\delta r n_{k}\right)^{s-2}=2^{s+2} \delta^{s-2} n_{k}^{s-1} r^{s}<\varepsilon r^{s}
\end{aligned}
$$

for sufficiently large $k$. Consequently,

$$
\lim _{r \downarrow 0} r^{-s} H^{s}(E \cap B(\mathbf{a}, r) \cap\{(x, y):|y-b| \geq \delta r\})=0,
$$

and $L$ is an approximate tangent line at $(a, b)$.

To prove that $E$ is not Lipschitz regular, we consider a rectifiable curve $C$ and show that $H^{s}(E \cap C)=0$. Suppose $H^{s}(E \cap C)>0$. Then by $2.1(3)$, there is $a \in E \cap C$ such that $\theta^{* s}(E \cap C, a)=\theta^{* s}(E, a)$. It follows that for arbitrarily large values of $k$ the point $a$ belongs to some $Q_{k, i}$ such that $C$ meets at least $n_{k}^{3} / 2$ squares $Q_{k+1, j}$ inside $Q_{k, i}$. Since the distance between any two such squares $Q_{k+1, j}$ is at least $d_{k} n_{k}^{-2} / 2$, we have for the length of $C$,

$$
H^{1}(C) \geq\left(n_{k}^{3} / 2\right)\left(d_{k} n_{k}^{-2} / 2\right)=n_{k} d_{k} / 4 .
$$

But $n_{k} d_{k} \rightarrow \infty$, which contradicts $H^{1}(C)<\infty$.

4. $(s, k)$ regular sets with positive lower density. In this section we establish the relations between the $(s, k)$ regularity concepts for an $s$-set $E$ under the additional hypothesis that $E$ has positive lower density almost everywhere.

4.1 TheOREM. Suppose $s \leq k, E \subset \mathbf{R}^{n}$ is $H^{s}$ measurable with $H^{s}(E)<\infty$, and $\theta_{*}^{s}(E, a)>0$ for $H^{s}$ almost all $a \in E$. Then

(1) If $s<k, E$ is $(s, k)$ Lipschitz regular.

(2) $E$ is $(s, k)$ approximately tangentially regular if and only if $E$ is $(s, k)$ tangentially regular.

(3) $E$ is $(s, k)$ approximately conically regular if and only if $E$ is $(s, k)$ conically regular.

After the proof we shall show that this theorem when combined with Theorem 3.9 gives all the valid implications. Thus, if $s<k$, in the case of positive lower density we are left with two different regularity concepts; tangential regularity implying conical regularity, while the third, Lipschitz, gives no additional regularity.

PROOF OF 4.1. Since by $2.1(1), \theta^{* s}(E, a) \leq 1$ for $H^{s}$ almost all $a \in E$, we can write $E$ as $E=\bigcup_{i=0}^{\infty} E_{i}$ with $H^{s}\left(E_{0}\right)=0$ and

$$
c_{i} r^{s} \leq H^{s}(E \cap B(a, r)) \leq d_{i} r^{s} \quad \text { for } 0<r \leq 1, a \in E_{i},
$$

where $c_{i}$ and $d_{i}$ are positive numbers independent of $a$ and $r$.

To prove (1) we show that if $s<k$, each $E_{i}$ is a Lipschitz image of a subset of $\mathbf{R}^{k}$. To do this we fix $i$, denote $F=E_{i}, c=c_{i}, d=d_{i}$, and we assume, as we clearly may, that $F \subset B(b, R)$ for some $b \in F, R \leq 1 / 2$. We shall first show that given $t$, $0<t<1, F$ can be covered with closed balls $B_{1}, \ldots, B_{N}$ of radius $t R$ such that $N \leq K t^{-s}$ where $K$ depends only on $c, d$, and $n$. To see this we use Besicovitch's covering theorem (see e.g. [G, $\S \mathrm{I} .1]$ ) to cover $F$ with balls $B_{1}, \ldots, B_{N}$ of radius $t R$ and centers in $F$ and such that for each $i$

$$
\sum_{i=1}^{N} \chi_{B_{i}} \leq M<\infty
$$


and

$$
H^{s}\left(E \cap B_{i}\right) \geq c t^{s} R^{s} \quad \text { for } i=1, \ldots, N .
$$

Here $M$ depends only on $n$ and $\chi_{B_{i}}$ denotes the characteristic function of $B_{i}$. Then

$$
\begin{aligned}
N c t^{s} R^{s} & \leq \sum_{i=1}^{N} H^{s}\left(E \cap B_{i}\right)=\int_{E} \sum_{i=1}^{N} \chi_{B_{i}} d H^{s} \\
& \leq M H^{s}(E \cap B(b, 2 R)) \leq M d 2^{s} R^{s} .
\end{aligned}
$$

Thus $N \leq K t^{-s}$ with $K=M d 2^{s} / c$.

Since the ball $B^{k}(R / 2)=\left\{x \in \mathbf{R}^{k}:|x| \leq R / 2\right\}$ contains the cube $\{x \in$ $\left.\mathbf{R}^{k}:\left|x_{i}\right| \leq R /(2 k)\right\}$, we can find at least $(5 k t)^{-k}$ points inside $B^{k}(R / 2)$ with mutual distances at least $5 t R$. We choose $t$, depending only on $c, d, k$, and $n$, so that $K t^{-s} \leq(5 k t)^{-k}$; that is, $t^{k-s} \leq(5 k K)^{-1}$. Then we can find balls $B_{1}^{\prime}, \ldots, B_{N}^{\prime} \subset B^{k}(R)$ of radius $t R$ such that the balls with same centers and radius $2 t R$ are disjoint. Define

$$
g: \bigcup_{i=1}^{N} B_{i}^{\prime} \rightarrow \bigcup_{i=1}^{N} B_{i}
$$

so that $g(x)=$ the center of $B_{i}$ for $x \in B_{i}^{\prime}$. If $x \in B_{i}^{\prime}, y \in B_{j}^{\prime}$, and $i \neq j$, then $|x-y| \geq t R$ and $|g(x)-g(y)| \leq 2 R$.

Therefore

$$
|g(x)-g(y)| \leq 2|x-y| / t
$$

for $x, y \in \bigcup_{i=1}^{N} B_{i}^{\prime}$; that is, $g$ is $2 / t$-Lipschitz.

Denote $g=f_{1}$. For each $i$ we then repeat the same construction with $F, B(b, R)$, $B^{k}(R), R, t R$ replaced by $F \cap B_{i}, B_{i}, B_{i}^{\prime}, t R, t^{2} R$ to obtain balls

$$
B_{i, 1}, \ldots, B_{i, N_{i}} \subset B_{i}, B_{i, 1}^{\prime}, \ldots, B_{i, N_{i}}^{\prime}, \quad N_{i} \leq K t^{-s},
$$

and a map $g_{i}: \bigcup_{j=1}^{N_{i}} B_{i, j}^{\prime} \rightarrow \bigcup_{j=1}^{N_{i}} B_{i, j}$ satisfying (5). Together these maps $g_{i}$ give us a $2 / t$-Lipschitz map

$$
f_{2}: \bigcup_{i, j} B_{i, j}^{\prime} \rightarrow \bigcup_{i, j} B_{i, j}
$$

Continuing in this manner we get a sequence $f_{i}$ of $2 / t$-Lipschitz maps whose restrictions to

$$
A=\bigcap_{m=1}^{\infty} \bigcup_{i_{1} \cdots i_{m}} B_{i_{1}, \ldots, i_{m}}^{\prime}
$$

converge to a $2 / t$-Lipschitz map $f: A \rightarrow \mathbf{R}^{n}$. Since $f(A)$ is compact, it follows $F \subset f(A)$, and the proof of $(1)$ is complete.

To prove (2), assume $E$ is $(s, k)$ approximately tangentially regular. Let $E_{i}, c_{i}$, and $d_{i}$ be as in (4). For each $i=1,2, \ldots$ we may assume that $E$ has an approximate tangent $k$-plane at each point of $E_{i}$. Let $a \in E_{i}$. Then there is $V \in G(n, k)$ such that for every $t, 0<t<1$,

$$
\lim _{r \downarrow 0} r^{-s} H^{s}\left(E \cap X\left(a, r, V^{\perp}, 1-t / 2\right)\right)=0 .
$$


Suppose $0<t<1, r>0$, and there is $b \in E_{i} \cap X\left(a, r, V^{\perp}, 1-t\right)$. Let $\rho=|a-b|$. Then for some $C>0$, independent of $a, b$, and $r$,

$$
B(b, C t \rho) \subset X\left(a, 2 \rho, V^{\perp}, 1-t / 2\right) .
$$

Consequently,

$$
c_{i}(C t \rho)^{s} \leq H^{s}(E \cap B(b, C t \rho)) \leq H^{s}\left(E \cap X\left(a, 2 \rho, V^{\perp}, 1-t / 2\right)\right) .
$$

For small enough $\rho$ this is impossible by (6), whence

$$
E_{i} \cap X\left(a, r, V^{\perp}, 1-t\right)=\varnothing
$$

for small enough $r$. It follows that $E$ is $(s, k)$ tangentially regular. Since the converse always holds, the proof of $(2)$ is complete.

The proof of (3) is a slight modification of the proof of (2), which we leave to the reader.

To complete the picture of Theorem 4.1 we need to show that positive lower density does not imply conical regularity and that positive lower density together with conical regularity do not imply tangential regularity. Both of these follow from the constructions in 5.3 and 5.4 .

4.2 Self-similar sets. Self-similar sets provide a great variety of sets with positive lower density. Suppose that a compact set $E \subset \mathbf{R}^{n}$ is self-similar satisfying the open set condition. This means that there are similarity maps $S_{i}: \mathbf{R}^{n} \rightarrow \mathbf{R}^{n}$, $i=1, \ldots, N, N \geq 2$, such that

$$
\left|S_{i}(x)-S_{i}(y)\right|=r_{i}|x-y| \quad \text { for } x, y \in \mathbf{R}^{n},
$$

where $0<r_{i}<1$, for which $E=\bigcup_{i=1}^{N} S_{i}(E)$ and that there is a nonempty open set $V$ for which

$$
\bigcup_{i=1}^{N} S_{i}(V) \subset V \quad \text { and } \quad S_{i}(V) \cap S_{j}(V)=\varnothing \quad \text { for } i \neq j .
$$

Let $s$ be the unique number satisfying $\sum_{i=1}^{N} r_{i}^{s}=1$. Hutchinson (see [H] or [FK]) showed that then $0<H^{s}(E)<\infty$ and there are $0<c, d<\infty$ such that

(4.3) $c r^{s} \leq H^{s}(E \cap B(a, r)) \leq d r^{s}$ for $a \in E, 0<r \leq 1$.

In particular, $\theta_{*}^{s}(E, a)>0$ for all $a \in E$.

Suppose $s<k$. According to 4.1(1) a self-similar set $E$ as above is always $(s, k)$ Lipschitz regular. It may be but need not be $(s, k)$ conically regular, as simple examples show. Due to [MP] it is $(s, k)$ tangentially regular if and only if it lies on some $k$-plane.

4.4 A construction of sets with positive lower density. Here we shall give a scheme, considerably more general than the self-similar construction, leading also to sets satisfying (4.3). Our proof below is based on the same ideas as those in [FK and $\mathbf{H}$ ], but avoiding the use of auxiliary measures it is somewhat more direct.

Suppose that $a, b, c, d$, and $s$ are positive real numbers, $s<n, m_{1}, m_{2}, \ldots$ are positive integers and that for every sequence of integers $i_{1}, \ldots, i_{k}, k=1,2, \ldots$, with $1 \leq i_{j} \leq m_{j}$, there correspond a compact set $E_{i_{1} \cdots i_{k}} \subset \mathbf{R}^{n}$, a closed ball $B_{i_{1} \cdots i_{k}}$, and a positive real number $d_{i_{1} \cdots i_{k}}$ such that the following conditions are satisfied for all $i_{1}, \ldots, i_{k}, 1 \leq i_{j} \leq m_{j}$ :

$$
E_{i_{1} \cdots i_{k} j} \subset E_{i_{1} \cdots i_{k}} \text { for all } j=1, \ldots, m_{k+1},
$$




$$
\begin{gathered}
B_{i_{1} \cdots i_{k}} \subset E_{i_{1} \cdots i_{k}}, \\
\operatorname{Int}\left(E_{i_{1} \cdots i_{k} i}\right) \cap \operatorname{Int}\left(E_{i_{1} \cdots i_{k} j}\right)=\varnothing \text { for } i \neq j, \\
a d\left(E_{i_{1} \cdots i_{k}}\right)<d\left(B_{i_{1} \cdots i_{k}}\right), \\
b d_{i_{1} \cdots i_{k}} \leq d\left(E_{i_{1} \cdots i_{k}}\right) \leq c d_{i_{1} \cdots i_{k}}, \\
\delta_{k}=\max \left\{d_{i_{1} \cdots i_{k}}: 1 \leq i_{j} \leq m_{j}, j=1, \ldots, k\right\} \rightarrow 0 \text { as } k \rightarrow \infty \\
\sum_{i_{1} \cdots i_{k}} \leq d d_{i_{1} \cdots i_{k} j} \text { for all } j=1, \ldots, m_{k+1}, \\
\sum_{j=1}^{m_{k+1}}\left(d_{i_{1} \cdots i_{k} j}\right)^{s}=\left(d_{i_{1} \cdots i_{k}}\right)^{s} .
\end{gathered}
$$

We define a compact set $E$ by

$$
E=\bigcap_{k=1}^{\infty} \bigcup_{i_{1} \cdots i_{k}} E_{i_{1} \cdots i_{k}} .
$$

It is clear from $[\mathbf{H}$ or $\mathbf{F K}]$ that the self-similar sets satisfying the open set condition are all included in this construction. Using (1)-(5) one readily sees that the sequence $m_{k}$ must be bounded; $\sup _{k} m_{k}<\infty$. In most examples $d_{i_{1} \cdots i_{k}}=$ $d\left(E_{i_{1} \ldots i_{k}}\right)$, but other applications are possible. Iterating (8) one obtains

$$
\sum_{j_{1} \cdots j_{l}}\left(d_{i_{1} \cdots i_{k} j_{1} \cdots j_{l}}\right)^{s}=\left(d_{i_{1} \cdots i_{k}}\right)^{s} .
$$

4.5 THEOREM. There exist positive and finite numbers $C$ and $D$ such that

$$
C r^{s} \leq H^{s}(E \cap B(x, r)) \leq D r^{s} \quad \text { for all } x \in E, 0<r \leq 1 .
$$

ProOF. Given $r>0$ we consider the following process. For each infinite sequence $j_{1}, j_{2}, \ldots, 1 \leq j_{i} \leq m_{i}$, let $k$ be the least integer with $d\left(E_{j_{1} \cdots j_{k}}\right)<r$. It exists since $d\left(E_{j_{1} \cdots j_{k}}\right) \rightarrow 0$ as $m \rightarrow \infty$. Then by (5) and (6) of 4.4

$$
\begin{aligned}
d\left(E_{j_{1} \cdots j_{k}}\right) & \geq b d_{j_{1} \cdots j_{k}} \geq b d^{-1} d_{j_{1} \cdots j_{k-1}} \\
& \geq b c^{-1} d^{-1} d\left(E_{j_{1} \cdots j_{k-1}}\right) \geq b c^{-1} d^{-1} r .
\end{aligned}
$$

Hence

$$
b c^{-1} d^{-1} r \leq d\left(E_{j_{1} \cdots j_{k}}\right)<r .
$$

Let $S_{r}$ be the set of all such sequences $j_{1} \cdots j_{k}$. Then the open $\operatorname{sets} \operatorname{Int}\left(E_{j_{1} \cdots j_{k}}\right)$, $\left(j_{1}, \ldots, j_{k}\right) \in S_{r}$, form a disjoint collection. Each $E_{j_{1} \cdots j_{k}}$ contains a ball $B_{j_{1} \cdots j_{k}}$ of diameter

$$
d\left(B_{j_{1} \cdots j_{k}}\right) \geq a d\left(E_{j_{1} \cdots j_{k}}\right) \geq a b c^{-1} d^{-1} r
$$

and is contained in a ball of radius $r$. Denoting $S_{r}(B)=\left\{j_{1} \cdots j_{k} \in S_{r}: B \cap\right.$ $\left.E_{j_{1} \cdots j_{k}} \neq \varnothing\right\}$, and $S(B)=S_{r}(B)$ if $r=d(B)$, we find (cf. [FK, Lemma 8.5]) that 
for any ball $B$ of diameter $r$ the set $S(B)$ contains at most $p$ elements where $p$ depends only on $a, b, c, d$, and $n$. Thus

$$
\sum_{j_{1} \cdots j_{k} \in S(B)} d\left(E_{j_{1} \cdots j_{k}}\right)^{s} \leq p d(B)^{s} .
$$

Let $\left\{A_{j}\right\}$ be a cover of $E$ such that $d\left(A_{j}\right) \leq r<1 / 2$ for $j=1,2, \ldots$ We may assume the sets $A_{j}$ are open, and since $E$ is compact we may reduce it to a finite cover $\{A\}_{j=1}^{m}$. For each $A_{j}$ there is a closed ball $B_{j}$ such that $d\left(B_{j}\right)=2 d\left(A_{j}\right) \leq 2 r$ and that $A_{j} \subset B_{j}$. Let $j_{0}$ be such that

$$
r_{0}=d\left(B_{j_{0}}\right)=\min \left\{d\left(B_{j}\right): j=1, \ldots, m\right\} .
$$

We shall see now that

$$
b^{s} c^{-s} \sum_{j_{1} \cdots j_{k} \in S_{r_{0}}\left(B_{j}\right)} d\left(E_{j_{1} \cdots j_{k}}\right)^{s} \leq \sum_{j_{1} \cdots j_{k} \in S\left(B_{j}\right)} d\left(E_{j_{1} \cdots j_{k}}\right)^{s} .
$$

The sequences of $S_{r_{0}}\left(B_{j}\right)$ are "longer" than those of $S\left(B_{j}\right)$ in the sense that if $j_{1} \cdots j_{k} \in S_{r_{0}}\left(B_{j}\right)$ there is $l \leq k$ such that $j_{1} \cdots j_{l} \in S\left(B_{j}\right)$. It follows from the definition of $S_{r}$ and 4.4(8) that the sum of the numbers $d_{j_{1} \cdots j_{k}}^{s}$ corresponding to a same $j_{1} \cdots j_{l}$ is at most $d_{j_{1} \cdots j_{l}}^{s}$. Hence (3) follows by $4.4(5)$.

From the relation between $A_{j}$ and $B_{j}$, and from (2) and (3) we obtain

$$
\begin{aligned}
2^{s} \sum_{j=1}^{m} d\left(A_{j}\right)^{s} & =\sum_{j=1}^{m} d\left(B_{j}\right)^{s} \geq p^{-1} \sum_{j=1}^{m} \sum_{j_{1} \cdots j_{k} \in S\left(B_{j}\right)} d\left(E_{j_{1} \cdots j_{k}}\right)^{s} \\
& \geq p^{-1} b^{s} c^{-s} \sum_{j=1}^{m} \sum_{j_{1} \cdots j_{k} \in S_{r_{0}}\left(B_{j}\right)} d\left(E_{j_{1} \cdots j_{k}}\right)^{s} \\
& \geq p^{-1} b^{s} c^{-s} \sum_{j_{1} \cdots j_{k} \in S_{r_{0}}} d\left(E_{j_{1} \cdots j_{k}}\right)^{s}
\end{aligned}
$$

where the last inequality is true because $\left\{B_{j}\right\}$ is a cover of $E$. Since

$$
\sum_{j_{1} \cdots j_{k}} d\left(E_{j_{1} \cdots j_{k}}\right)^{s} \geq b^{s} c^{-s} d\left(E_{1}\right)^{s}
$$

we have

$$
H^{s}(E) \geq p^{-1} b^{2 s} c^{-2 s} d\left(E_{1}\right)^{s} .
$$

Let $x \in E$ and $0<r \leq 1$. To prove the required inequalities we may assume $r<d\left(E_{1}\right)$. Then there is $j_{1} \cdots j_{k} \in S_{r}$ such that $x \in E_{j_{1} \cdots j_{k}}$. Since $d\left(E_{j_{1} \cdots j_{k}}\right)<r$, we have $E_{j_{1} \cdots j_{k}} \subset B(x, r)$ and, applying (4) to the set $E \cap E_{j_{1} \cdots j_{k}}$,

$$
H^{s}(E \cap B(x, r)) \geq H^{s}\left(E \cap E_{j_{1} \cdots j_{k}}\right) \geq p^{-1} b^{2 s} c^{2 s} d\left(E_{j_{1} \cdots j_{k}}\right)^{s} .
$$

Finally by (1)

$$
H^{s}(E \cap B(x, r)) \geq p^{-1} b^{2 s} c^{-2 s} b^{s} c^{-s} d^{-s} r^{s}=C r^{s},
$$

with $C=p^{-1} b^{3 s} c^{-3 s} d^{-s}$, which gives our first inequality.

In a similar way we obtain

$$
H^{s}(E \cap B(x, r)) \leq \sum_{j_{1} \cdots j_{k} \in S(B(x, r))} d\left(E_{j_{1} \cdots j_{k}}\right)^{s} \leq p 2^{s} r^{s},
$$

and the second inequality follows with $D=p 2^{s}$. 
5. Projection properties of $(s, k)$ regular sets. In this section we answer the following question: Suppose $E \subset \mathbf{R}^{n}$ is an $s$-set. Which of the $(s, k)$ regularity properties of $E$ imply that $H^{s}\left(P_{V} E\right)>0$ for $V_{n, k}$ almost all $V \in G(n, k)$ or that the set of such $k$-planes $V$ has positive $V_{n, k}$ measure? Recall from [FK, $\left.\S 6.3\right]$ that $\operatorname{dim} P_{V} E$ always equals $s$ for $\mathcal{V}_{n, k}$ almost all $V \in G(n, k)$.

5.1 ThEOREM. Suppose $E \subset \mathbf{R}^{n}$ is $H^{s}$ measurable with $0<H^{s}(E)<\infty$.

(1) If $E$ is $(s, k)$ conically regular, then

$$
V_{n, k}\left\{V: H^{s}\left(P_{V} E\right)>0\right\}>0 .
$$

(2) If $E$ is $(s, k)$ tangentially regular, then $H^{s}\left(P_{V} E\right)>0$ for $\mathcal{V}_{n, k}$ almost all $V \in G(n, k)$.

After the proof we shall construct examples to show that this theorem gives a complete answer to the above question.

PROOF. If $E$ is $(s, k)$ conically regular, then there are $F \subset E$ with $H^{s}(F)>0$, $0<t<1$, and $W \in G(n, k)$ such that

$$
F \cap X\left(a, \infty, W^{\perp}, t\right)=\varnothing \quad \text { for } a \in F .
$$

Furthermore, if $E$ is $(s, k)$ tangentially regular, we can let $t$ be as close to one as we please (with $F$ and $W$ depending on $t$ ). From (2.3) we infer

$$
F \cap X\left(a, \infty, V^{\perp}, u\right)=\varnothing
$$

whenever $V \in G(n, k)$ with $\left\|P_{V}-P_{W}\right\|<t-u, 0<u<t$. As in the proof of 3.9(4), this means that $P_{V} \mid F$ has Lipschitz inverse. Since Lipschitz maps preserve sets of $H^{s}$ measure zero, $H^{s}\left(P_{V} F\right)>0$ for any $V$ with $\left\|P_{V}-P_{W}\right\|<t-u$. As an open subset of $G(n, k),\left\{V:\left\|P_{V}-P_{W}\right\|<t-u\right\}$ has positive $\mathcal{V}_{n, k}$ measure, and (1) follows.

If $E$ is $(s, k)$ tangentially regular, given $\varepsilon, 0<\varepsilon<1$, we can choose $t$ and $u$ such that $t-u=1-\varepsilon$, and we find that

$$
\nu_{n, k}\left\{V: H^{s}\left(P_{V} E\right)=0\right\} \leq \mathcal{V}_{n, k}\left\{V:\left\|P_{V}-P_{W}\right\| \geq 1-\varepsilon\right\} .
$$

It suffices to show that the right-hand side, which is independent of $W$, tends to zero as $\varepsilon \rightarrow 0$. This follows once we know that $\mathcal{V}_{n, k}\left\{V:\left\|P_{V}-P_{W}\right\|=1\right\}=0$. Since $\left\|P_{V}-P_{W}\right\|=1$ if and only if $V \cap W^{\perp} \neq\{0\}$, the following lemma completes the proof.

5.2 LEMMA. Let $k$ and $m$ be integers such that $0 \leq k \leq n-1,1 \leq m \leq n-1$, $k+m \leq n$, and let $W \in G(n, m)$. Then, with $G(n, 0)=\{0\}$,

$$
\mathcal{V}_{n, k}\{V \in G(n, k): V \cap W \neq\{0\}\}=0 \text {. }
$$

PROOF. The lemma is obvious for $n=2$. We proceed by induction on $n$. Suppose the lemma holds for $n-1$. We may assume $k \geq 1$. For any Borel set $A \subset G(n, k)$,

$$
\mathcal{V}_{n, k}(A)=\int \mathcal{V}_{L^{\perp}, k-1}\left\{U \subset L^{\perp}: L+U \in A\right\} d \mathcal{V}_{n, 1} L
$$

where $\mathcal{V}_{L^{\perp}, k-1}$ is the invariant measure on the Grassmannian of all linear $(k-1)$ subspaces of $L^{\perp}$. This identity follows from the uniqueness of $V_{n, k}$, since the right-hand side defines a rotationally invariant measure. Evidently,

$$
\mathcal{V}_{n, 1}\{L \in G(n, 1): L \subset W\}=0,
$$


and thus the integration in the above formula can be performed over the lines $L$ with $L \not \subset W$. For any such $L$, the conditions $(L+U) \cap W \neq\{0\}$ and $U \subset L^{\perp}$ imply

$$
L^{\perp} \cap(W+L) \cap U=(W+L) \cap U \neq\{0\} .
$$

Hence by the induction hypothesis

$$
\mathcal{V}_{L^{\perp}, k-1}\{U:(L+U) \cap W \neq\{0\}\} \leq \mathcal{V}_{L^{\perp}, k-1}\left\{U: L^{\perp} \cap(W+L) \cap U \neq\{0\}\right\}=0 .
$$

Integrating over the lines $L$ with $L \not \subset W$ and taking

$$
A=\{V \in G(n, k): V \cap W \neq\{0\}\},
$$

we obtain the desired result.

5.3. For $0<s<1$ we construct a compact s-set $E \subset \mathbf{R}^{2}$ with positive lower density such that $H^{s}\left(P_{L} E\right)=0$ for all $L \in G(2,1)$. Thus $E$ is $(s, 1)$ Lipschitz regular but not $(s, 1)$ approximately conically regular.

Let $s$ be a real number, $0<s<1$. We consider in $\mathbf{R}^{2}$ the unit closed disc $C_{0}=\left\{x \in \mathbf{R}^{2}:|x| \leq 1\right\}$. Let $d_{0}$ be the horizontal direction and $d_{1}$ the direction such that the angle

$$
\left({\widehat{d_{0}, d_{1}}}_{1}=\alpha_{1}=\pi / 4 .\right.
$$

Inside $C_{0}$ and with centers in the diameter of $C_{0}$ in the direction $d_{1}$ we construct the disjoint closed discs $C_{1}, C_{2}, C_{3}$, symmetrically distributed along such diameter, as we can see in Figure 2, and with radius $r$ such that $3 \cdot r^{s}=1$. (Observe that since $s<1$, then $r<\frac{1}{3}$ and that construction is possible.)

We continue in this way and we suppose that we have constructed the $3^{k}$ discs $C_{j_{1} \cdots j_{k}}, 1 \leq j_{i} \leq 3$ for $i=1,2, \ldots, k$. Inside every disc $C_{j_{1} \cdots j_{k}}$ we construct the disjoint closed discs $C_{j_{1} \cdots j_{k, i}}, i=1,2,3$, of radius $r^{k+1}$ and with centers in the diameter of $C_{j_{1} \ldots j_{k}}$ in the direction $d_{k+1}$ which verifies

$$
\left(d_{k}, \widehat{d}_{k+1}\right)=\alpha_{k+1}=\frac{\pi}{4 \cdot(k+1)} .
$$

We denote $S_{k}=\left\{j_{1} \cdots j_{k}: 1 \leq j_{i} \leq 3\right.$ for $\left.i=1,2, \ldots, k\right\}$ and define the set

$$
E=\bigcap_{k=1}^{\infty} \bigcup_{j_{1} \cdots j_{k} \in S_{k}} C_{j_{1} \cdots j_{k}}
$$

which is a compact $s$-set and with positive lower density. We shall show now that $H^{s}\left(P_{L} E\right)=0$ for all $L \in G(2,1)$. Let $L \in G(2,1)$ be a line in $\mathbf{R}^{2}$ and $d_{L}$ the direction perpendicular to $L$.

We fix an arbitrary positive integer $k$. We choose first $\alpha, 0<\alpha<\pi$, such that

$$
\sin \alpha<r^{k},
$$

and then a positive integer $p$ such that

$$
\sum_{j=p}^{p+k+1} \alpha_{j}<\alpha
$$

which is always possible because $\alpha_{j} \rightarrow 0$ as $j \rightarrow \infty$. Then there is an integer $k_{0} \geq p$ such that

$$
\left(d_{k_{0}+1}, d_{L}\right) \leq \alpha \text { and }\left(d_{k_{0}}, d_{L}\right)>\alpha
$$




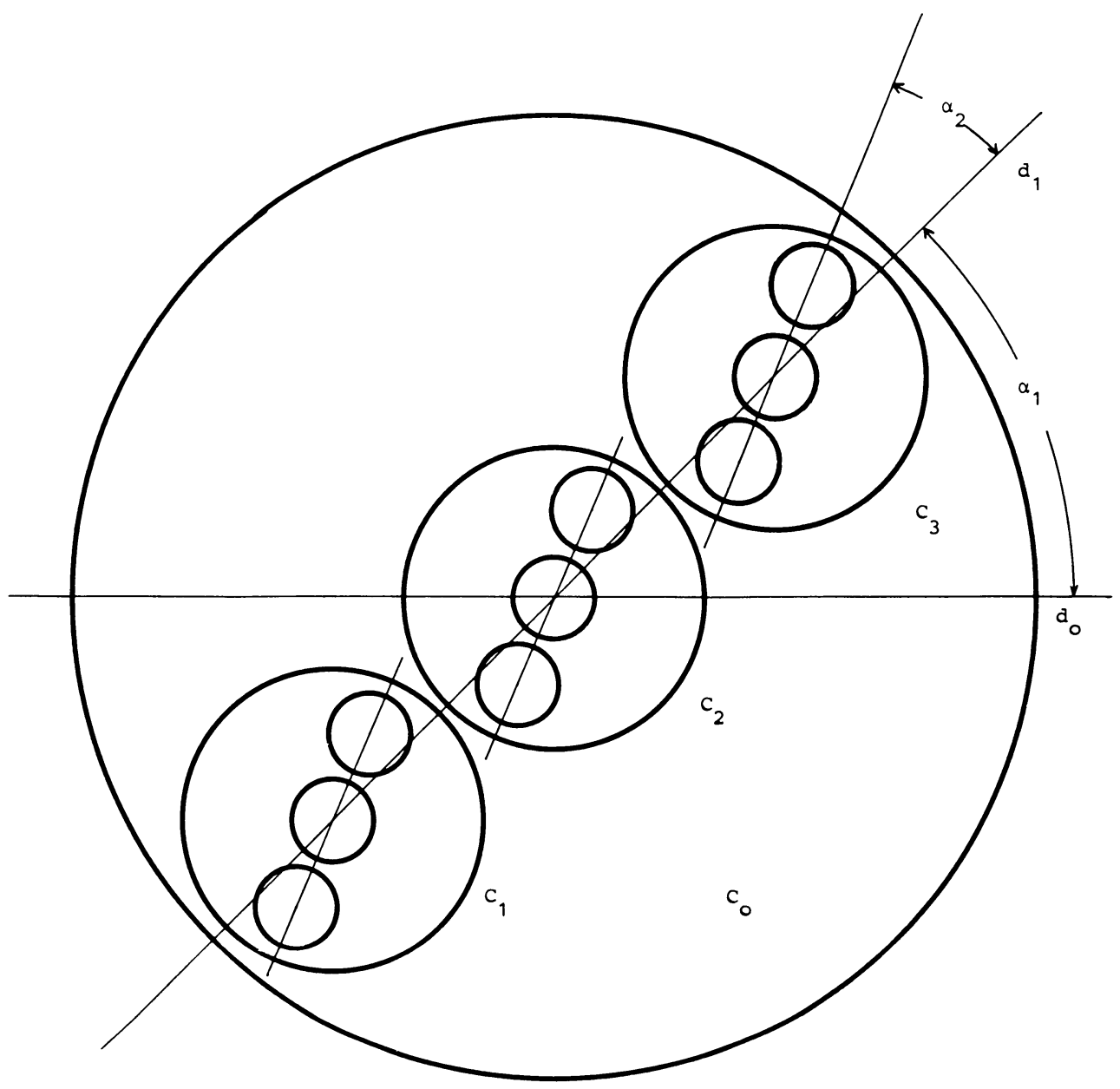

FIGURE 2

and since $\left\{\alpha_{j}\right\}$ is a decreasing sequence, we also have

$$
\sum_{j=k_{0}}^{k_{0}+k+1} \alpha_{j}<\alpha
$$

From the construction of $E$ we may deduce that for each $j_{1} \cdots j_{k_{0}} \in S_{k_{0}}$ the centers of the discs $C_{j_{1} \cdots j_{k_{0}} \cdots j_{k_{0}+k}}$ of the stage $k_{0}+k$ have distances from the diameter of $C_{j_{1} \cdots j_{k_{0}}}$ in the direction $d_{L}$ less than $r^{k_{0}} \sin \alpha$ (see Figure 3) and since such discs have radius $r^{k_{0}+k}$, inequality (1) shows us that the $\operatorname{discs} C_{j_{1} \cdots j_{k_{0}+1} \cdots j_{k_{0}+k}}$ all meet the diameter of $C_{j_{1} \cdots j_{k_{0}}}$ in the direction $d_{L}$. Then, if for $j_{1} \cdots j_{k_{0}} \in S_{k_{0}}$ we denote

$$
I_{j_{1} \cdots j_{k_{0}}}^{k}=P_{L}\left(\bigcup_{j_{k_{0}+1} \cdots j_{k_{0}+k} \in S_{k}} C_{j_{1} \cdots j_{k_{0}+1} \cdots j_{k_{0}+k}}\right)
$$

this projection is an interval, and since

$$
d\left(I_{j_{1} \cdots j_{k_{0}}}^{k}\right) \leq 2 \cdot d\left(C_{j_{1} \cdots j_{k_{0}+k}}\right) \quad \text { for all } j_{1} \cdots j_{k_{0}+k} \in S_{k_{0}+k}
$$




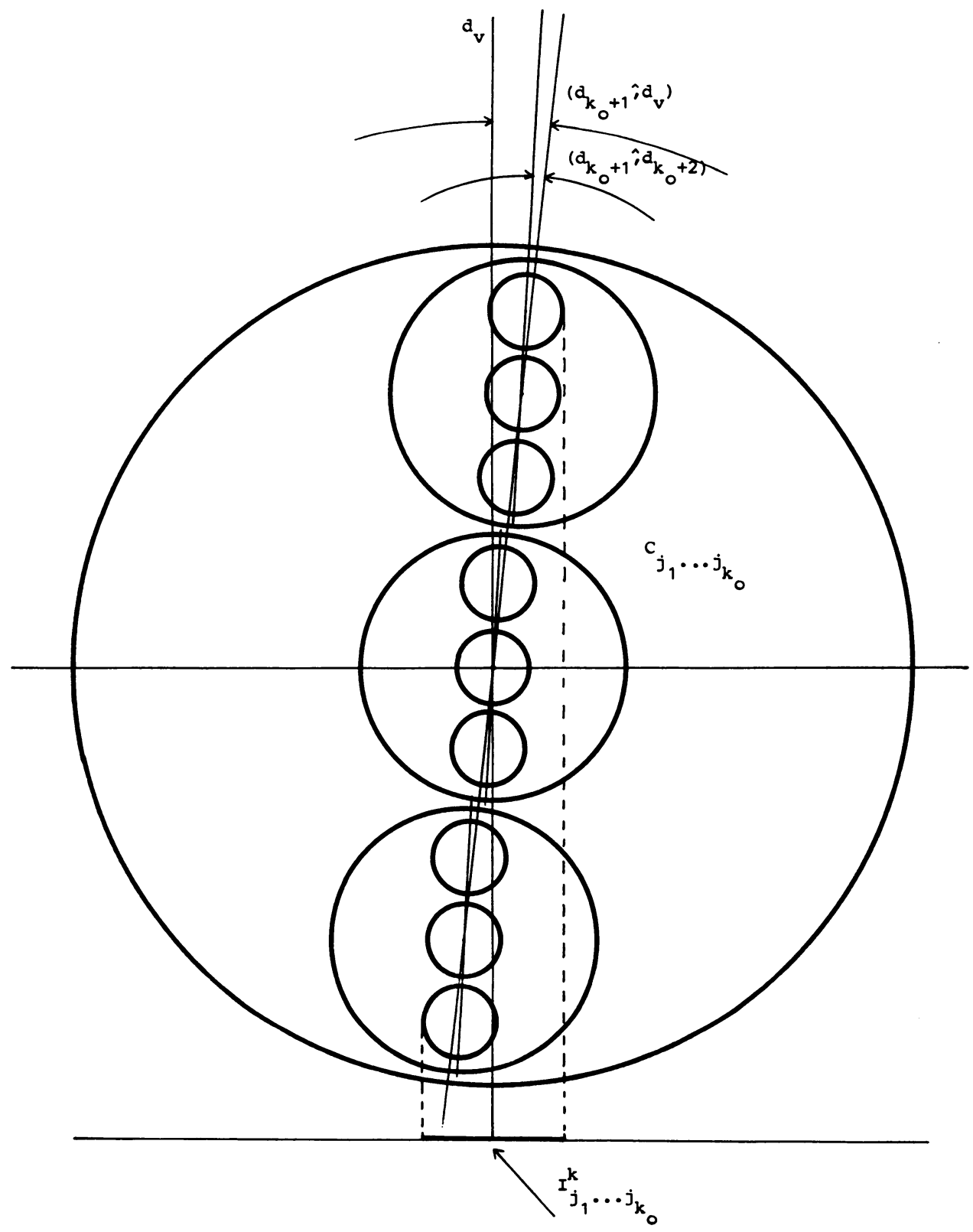

FIGURE 3

we have

(4)

$$
\begin{aligned}
\sum_{j_{1} \cdots j_{k_{0}} \in S_{k_{0}}} d\left(I_{j_{1} \cdots j_{k_{0}}}^{k}\right)^{s} & \leq \frac{2^{s}}{3^{k}} \sum_{j_{1} \cdots j_{k_{0}+k} \in S_{k_{0}+k}} d\left(C_{j_{1} \cdots j_{k_{0}+k}}\right)^{s} \\
& =\frac{2^{s}}{3^{k}} \cdot 2^{s}=\frac{2^{2 s}}{3^{k}}
\end{aligned}
$$


because $3 r^{s}=1$ implies $\sum_{i=1}^{3} d\left(C_{j_{1} \cdots j_{k, i}}\right)^{s}=d\left(C_{j_{1} \cdots j_{k}}\right)^{s}$, and $d\left(C_{0}\right)^{s}=2^{s}$. But the collection $\left\{I_{j_{1} \cdots j_{k_{0}}}^{k}\right\}_{j_{1} \cdots j_{k_{0}} \in S_{k_{0}}}$ is a $\delta$-cover of $P_{L} E, \delta=4 r^{k_{0}+k}$, and so

$$
H_{\delta}^{s}\left(P_{L} E\right) \leq \frac{2^{2 s}}{3^{k}}
$$

Since $k$ is arbitrarily large we obtain $H^{s}\left(P_{L} E\right)=0$ which gives our assertion.

5.4 For $0<s<1$ there is a compact $(s, 1)$ conically regular $s$-set $E \subset \mathbf{R}^{2}$ such that $\theta_{*}^{s}(E, a)>0$ for $a \in E$ and $\mathcal{V}_{2,1}\left\{L: H^{s}\left(P_{L} E\right)=0\right\}>0$. Thus $E$ is not $(s, 1)$ approximately tangentially regular.

As in 5.3 we consider the unit closed disc

$$
C_{0}=\left\{x \in \mathbf{R}^{2}:|x| \leq 1\right\}
$$

and the sequence $\left\{\alpha_{j}\right\}_{j=1}^{\infty}$ defined by $\alpha_{j}=\pi / 4 j$.

Let $d$ and $d^{\prime}$ be the directions such that $(\widehat{H, d})=\pi / 4$ and $\left(\widehat{H, d^{\prime}}\right)=3 \pi / 4$, where $H$ is the direction of the horizontal axis, as we see in Figure 4.

Let $k_{0}=0$ and for $n=1,2, \ldots$ let $\alpha_{k_{n}}$ be such that

$$
\sum_{j=k_{n-1}+1}^{k_{n}} \alpha_{j} \leq \frac{\pi}{2} \text { and } \sum_{j=k_{n-1}}^{k_{n}+1} \alpha_{j}>\frac{\pi}{2} .
$$

We define a sequence of directions in the following way:

(a) $d_{1}$ is the direction such that $\left(\widehat{d, d}_{1}\right)=\alpha_{1}$,

(b) $d_{k_{n}+1}$ is the direction $d\left(d_{k_{n}+1} \equiv d\right)$ for all $n=1,2, \ldots$,

(c) For each $n=1,2, \ldots$ and for $j \neq k_{n}+1, d_{j}$ is the direction which verifies $\left(d_{j-1}, d_{j}\right)=\alpha_{j}$.

We construct now a compact $s$-set $E \subset \mathbf{R}^{2}(0<s<1)$ with positive lower density, in the same way as that of 5.3, distributing the consecutive discs in an analogous way but with centers in the diameters in the consecutive directions of the sequence $d_{j}$ constructed above.

In an identical way as in 5.3 we show that $H^{s}\left(P_{L} E\right)=0$ for all lines $L \in G(2,1)$ whose direction is between $d$ and $d^{\prime}$; that is, such that

$$
\left(\widehat{d_{L}, d}\right) \geq 0 \text { and }\left(\widehat{L_{L}, d^{\prime}}\right) \leq \pi / 2
$$

Then $E$ verifies that

$$
\mathcal{V}_{2,1}\left\{L \in G(2,1): H^{s}\left(P_{L} E\right)=0\right\}>0 .
$$

Note finally that the selection of $d$ and $d^{\prime}$ is irrelevant and the important thing is that $\left(\widehat{d, d^{\prime}}\right)>0$ to get the above result.

On the other hand this set is clearly $(s, 1)$ conically regular since for $t<\sqrt{2} / 2$ we have

$$
X(a, \infty, H, t) \cap E=\varnothing .
$$

5.5 For $0<s<1$ we construct a compact $(s, 1)$ approximately tangentially regular s-set $E \subset \mathbf{R}^{2}$ such that $H^{s}\left(P_{L} E\right)=0$ for all $L \in G(2,1)$.

We construct $E$ so that the $x$-axis is an approximate tangent line for $E$ at all of its points. Let $Q$ be a closed square in $\mathbf{R}^{2}$ with sides parallel to the coordinate axis, side-length $d$, and center $(a, b)$. Given positive integers $m$ and $n$, and $\theta \in[0, \pi / 2)$ we first describe an operation to generate a disjoint collection of subsquares of $Q$. 


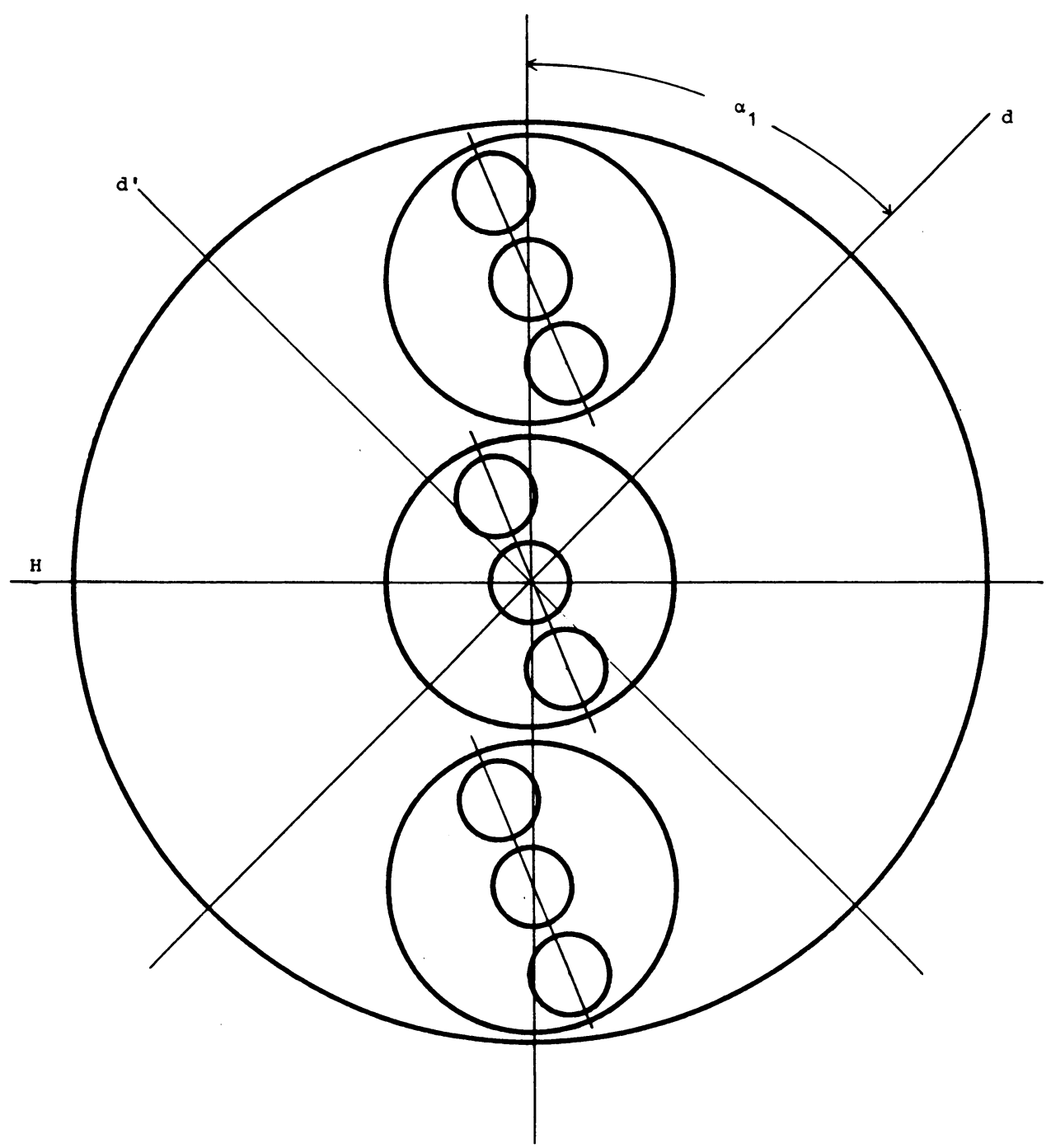

FiguRE 4

Let $R$ be (Figure 5) the rectangle $\{(x, y):|x-a| \leq d / 2,|y-b| \leq d /(2 n)\}$ contained in $Q$. Divide $R$ into squares $Q_{1}, \ldots, Q_{n}^{\prime}$ of side-length $d / n$. Let $Q_{i}$ be the square with the same center as $Q_{i}^{\prime}$, side-length $d /(2 n)$, and two sides making an angle $\theta$ with the $x$-axis. Partition each $Q_{i}$ into $m^{2}$ squares $P_{i j}^{\prime}$ of side-length $d /(2 m n)$. Finally let $P_{i j}$ be the square with sides parallel to the axis which has the same center as $P_{i j}^{\prime}$ and side-length $d^{\prime}$ with $d^{\prime}$ defined by $m^{2} n d^{\prime s}=d^{s}$. We define

$$
\alpha=\alpha(m, n)=\overline{\operatorname{arc}} \tan \left(\frac{d^{\prime}}{d /(2 n)}\right)=\overline{\operatorname{arc}} \tan \left(2 m^{-2 / s} n^{1-1 / s}\right),
$$

and observe that if $\theta \leq \phi \leq \theta+\alpha$ or $\theta+\pi / 2 \leq \phi \leq \theta+\pi / 2+\alpha$ then there are intervals

$$
I_{i k} \subset L_{\phi}=\{t(\cos \phi, \sin \phi): t \in R\}, \quad k=1, \ldots, m, i=1, \ldots, n,
$$




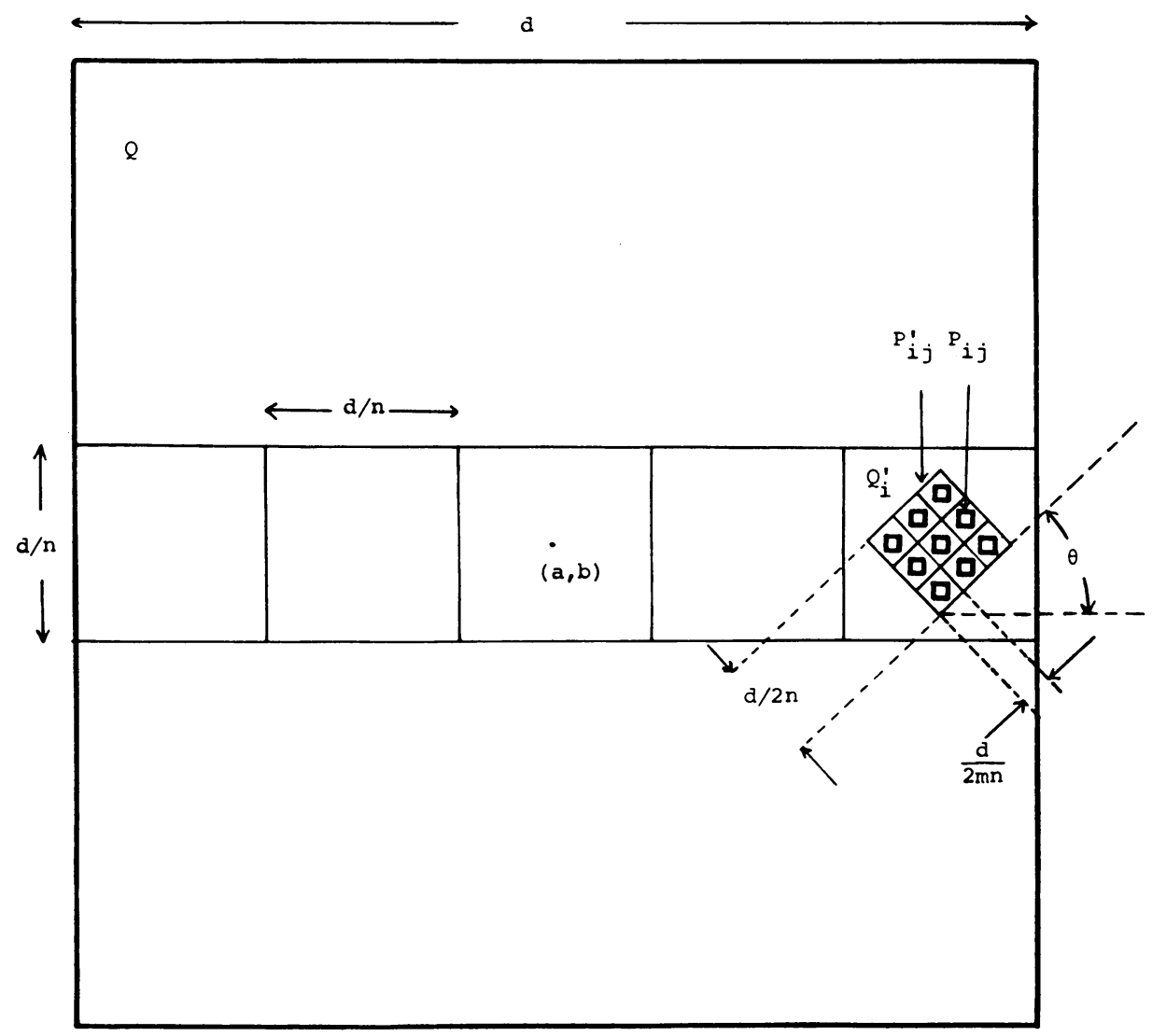

FIGURE 5

such that

$$
P_{L_{\phi}}\left(\bigcup_{j=1}^{m^{2}} P_{i j}\right) \subset \bigcup_{k=1}^{m} I_{i k},
$$

$d\left(I_{i k}\right)=2 d^{\prime}$, whence $\sum_{i, k} d\left(I_{i k}\right)^{s}=(2 d)^{s} / m$.

Choose strictly increasing sequences $m_{k}$ and $n_{k}$ of positive integers, and let $\alpha_{k}=\alpha\left(m_{k}, n_{k}\right)$. Starting from the square $Q_{1,1}=\left[0, d_{1}\right] \times\left[0, d_{1}\right]$, perform the above operation with $m=m_{1}, n=n_{1}, \theta=0$. In each of the squares $P_{i j}$ thus obtained perform the operation with $m=m_{1}, n=n_{1}, \theta=\alpha_{1}$. Continue this with $m=m_{1}, n=n_{1}, \theta=j \alpha_{1}$ for $j=2,3, \ldots$ until $\pi / 2 \leq j \alpha_{1}$. Let $Q_{2,1}, \ldots, Q_{2, p_{1}}$ be all the subsquares of $Q$ obtained at the last step. Their common side-length $d_{2}$ satisfies $p_{1} d_{2}^{s}=d_{1}^{s}$. Moreover for every $\phi \in[0, \pi)$ there are intervals $I_{j} \subset L_{\phi}$ such that

$$
\begin{aligned}
& P_{L_{\phi}}\left(\bigcup_{i} Q_{2, i}\right) \subset \bigcup_{j} I_{j}, \\
& \sum_{j} d\left(I_{j}\right)^{s} \leq\left(2 d_{1}\right)^{s} / m_{1} .
\end{aligned}
$$


Next in each square $Q_{2, i}$ we perform the similar sequence of operations with parameters $m_{2}, n_{2}, j \alpha_{2}$ with $j=0,1,2, \ldots$ until $j \alpha_{2} \geq \pi / 2$ obtaining the squares $Q_{3, j}$. Continuing in this manner we get squares $Q_{k, j}, j=1, \ldots, p_{k}, k=1,2, \ldots$, and define

$$
E=\bigcap_{k=1}^{\infty} \bigcup_{j=1}^{p_{k}} Q_{k, j} .
$$

Then $0<H^{s}(E)<\infty$, and relations analogous to (1) yield $H^{s}\left(P_{L} E\right)=0$ for all $L \in G(2,1)$. Finally that the $x$-axis is an approximate tangent line for $E$ at all of its points follows as in 3.13 .

\title{
REFERENCES
}

[FK] K. J. Falconer, Geometry of fractal sets, Cambridge Univ. Press, 1985.

[FH] H. Federer, Geometric measure theory, Springer-Verlag, 1969.

[G] M. de Guzmán, Differentiation of integrals in $R^{n}$, Springer-Verlag, 1975.

[H] J. E. Hutchinson, Fractals and self-similarity, Indiana Univ. Math. J. 30 (1981), 713-747.

[MB] B. B. Mandelbrot, The fractal geometry of nature, Freeman, 1982.

[MM] M. A. Martin, Propiedades de proyección de fractales, Tesis Doctoral, Universidad Complutense, Madrid, 1986.

[MJ] J. M. Marstrand, The $(\phi, s)$ regular subsets of $n$-space, Trans. Amer. Math. Soc. 113 (1964), 369-392.

[MP] P. Mattila, On the structure of self-similar fractals, Ann. Acad. Sci. Fenn. Ser. A I 7 (1982), 189-195.

[P] D. Preiss, Geometry of measures in $R^{n}$, distribution, rectifiability, and densities, Ann. of Math. 125 (1987), 537-643.

[R] M. Ross, Federer's structure theorem, Research Report, Centre for Mathematical Analysis, Australian National University, 1984.

[S] A. Salli, Upper density theorems for Hausdorff measures on fractals, Dissertationes Ann. Acad. Sci. Fenn. Ser. A I 55 (1985).

[W] B. White, Problem 3.10, Proc. Sympos. Pure Math., vol. 44, Amer. Math. Soc., Providence, R. I., 1985, p. 447.

\author{
Department of Mathematics, Universidad autónoma, Madrid, Spain \\ Department of Mathematics, University of Helsinki, Helsinki, Finland
}

